\title{
Lipödem - Mythen und Fakten Teil 1
}

\author{
T. Bertsch ${ }^{1} ;$ G. Erbacher ${ }^{1,2}$ \\ ${ }^{1}$ Földiklinik Hinterzarten - Europäisches Zentrum für Lymphologie; ${ }^{2}$ Dipl.-Psychologin, Psychologische Psychothera- \\ peutin, Supervisorin (hsi)
}

\section{Schlüsselwörter}

Lipödem, Progredienz, Adipositas, Psychische Erkrankung, Wissenschaftliche Evidenz

\section{Zusammenfassung}

Das Lipödem ist weit mehr als einfach nur dickere und schmerzhafte Beine! Die Erkrankung Lipödem ist mit zahlreichen Mythen behaftet. Im ersten Teil dieser Übersicht werfen wir einen kritischen Blick auf zwei populäre Statements zum Lipödem; Statements, die vor Jahrzehnten schon Eingang in wissenschaftliche Publikationen gefunden haben und seither unkritisch und stetig wiederholt werden; Statements, die inzwischen zum selbstverständlichen Wissensallgemeingut von Lipödempatientinnen und vor allem auch von Lipödem-Selbsthilfegruppen geworden sind. In unserer Darstellung über die Mythen des Lipödems fokussieren wir uns in diesem Beitrag vor allem auf zwei Aspekte, die aufs Engste mit dem Lipödem verbunden sind: auf die Adipositas sowie auf die psychische Situation von Lipödempatientinnen - die wiederum eng mit der Adipositas in Zusammenhang steht. Dabei überprüfen wir zwei häufig publizierte Statements auf wissenschaftliche Evidenz: 1. „Das Lipödem ist eine progrediente Erkrankung", 2. „Ein Lipödem macht psychisch krank". Beide Statements widersprechen in hohem Maße unserer seit Jahren bestehenden täglichen klinischen Erfahrung mit diesem speziellen Patientengut. Gleichzeitig haben wir im Rahmen unserer umfang-

Korrespondenzadresse

Dr. med. Tobias Bertsch

Leitender Oberarzt

Földiklinik GmbH \& Co.KG

Rösslehofweg 2-6

79856 Hinterzarten

Tel. +4976521240

Fax +497652 124116

E-Mail: tobias.bertsch@foeldiklinik.de reichen Literaturrecherche festgestellt, dass es auch keine Evidenz für diese in den „Lipödemsprachgebrauch" eingegangenen Behauptungen gibt. Tatsachlich ist das Lipödem in der Regel keine progrediente Erkrankung! Vielmehr liegt bei Lipödempatientinnen häufig eine Gewichtsprogredienz (meist eine Adipositasprogredienz) vor, in deren Folge sich auch das Lipödem verschlechtern kann. Unsere Pilotstudie zum 2. Statement macht deutlich, dass in der Regel nicht das Lipödem Ursache von psychischen Erkrankungen ist. Hier weisen unsere Ergebnisse in die umgekehrte Richtung: Eine vorbestehende - psychische Vulnerabilität kann ganz wesentlich zum Krankheitsbild Lipödem beitragen. Um das Lipödem in seiner ganzen Komplexität und Vielfalt zu erfassen, braucht es mehr als nur Medizin. Psychosoziale Therapieansätze sollten integraler Bestandteil eines wirksamen multimodalen Behandlungskonzepts sein. Neben den beiden dargestellten Mythen gibt es weitere, die sich um das Lipödem ranken. Diese werden in weiteren Ausgaben dieser Zeitschrift diskutiert werden.

\section{Keywords}

Lipoedema, Progressive, Obesity, Mental Disease, Scientific Evidence

\section{Summary}

Lipoedema is much more than just fat and painful legs! Lipoedema as a disease is associ-

Lipoedema - myths and facts Part 1

Phlebologie 2018; 47: 84-92

https://doi.org/10.12687/phleb2411-2-2018

Eingereicht: 30. Januar 2018

Angenommen: 25. Februar 2018

English version available at:

www.phlebologieonline.de ated with numerous myths. In this overview we take a critical view of two popular statements on lipoedema. We have found that statements made in scientific publications decades ago have been repeated over and over again without criticism. These statements have become part of the general knowledge for lipoedema patients and lipoedema self-help groups. In our representation of the myths regarding lipoedema, we will focus in this contribution on two aspects in particular, which are closely associated with lipoedema: on obesity and the psychiatric health of lipoedema patients, which is again closely associated with obesity. We will thus examine two commonly publicised statements for actual scientific evidence: 1 . "Lipoedema is a progressive disease", 2. „Lipoedema causes mental diseases". Both statements contradict to a high degree our daily clinical experience with this specific subset of patients. We simultaneously established, through extensive literature research, that there was no evidence for these statements which have become part of the vocabulary for lipoedema. In fact not lipoedema is progressiv! The truth is that lipoedema patients often experience weight progression (mostly a progression in obesity), which leads to worsening of the lipoedema as a result. Our pilot study on the second statement shows that lipoedema is generally not the cause of mental illness. In fact, our studies show the opposite: an existing psychological vulnerability can contribute signficantly to the disease lipoedema. In order to encompass lipoedema in all of its complexity and variety we need more than just medicine. Psychosocial therapy approaches should be an integral component of an effective multimodal treatment concept. 


\section{Einleitung}

Das Lipödem ist weitaus mehr als dickere, schmerzhafte Beine!

Allerdings - auch das sei betont - nicht jedes dickere Bein ist ein Lipödem (1)!

In der Földiklinik in Hinterzarten - Europäisches Zentrum für Lymphologie - behandeln wir jedes Jahr ca. 3000 Patientinnen mit der Diagnose Lipödem - ambulant und stationär. Die überwältigende Mehrheit dieser Patientinnen kommt mit Vorstellungen und Erwartungen in unsere Klinik, die durch Medien aber auch durch Ärzte genährt wurden -Vorstellungen über das Lipödem, die weit entfernt sind von wissenschaftlicher Evidenz, Erwartungen, die oft jenseits des Machbaren liegen.

Ein wesentliches Grundproblem dieser Erkrankung besteht darin, dass das Lipödem keine objektiven Diagnosekriterien aufweist, dass kein Parameter, kein Laborwert, keine Bildgebung existiert, mit der man diese Erkrankung auch nur annährend zweifelsfrei diagnostizieren kann. Entsprechend der Deutschen LipödemLeitlinie von 2015 erfolgt die „Diagnosestellung des Lipödems durch Anamnese, Inspektion und Palpation anhand der typischen Charakteristika“. Diese Charakteristika lassen sich auf vier Symptome herunterbrechen:

- disproportionale Fettgewebsvermehrung an Beinen (und/oder Armen)

- Schweregefühl und/oder Schmerzhaftigkeit der betroffenen Extremitäten

- Hämatomneigung

- Ödeme - im Tagesverlauf zunehmend (2).

Dieser Mangel an objektiven und klaren Diagnosekriterien führt auch dazu, dass keine verlässlichen Zahlen zur Prävalenz des Lipödems existieren. Weit überwiegend wird die Diagnose Lipödem von lymphologisch unerfahrenen Kollegen und fast noch häufiger von den Patientinnen selbst gestellt. In unserem Patientengut ist die Diagnose Lipödem (und noch häufiger die Diagnose "Lipolymphödem") die mit Abstand häufigste Fehldiagnose, die wir täglich sehen. Alle - auch in wissenschaftlichen Publikationen - kursierenden Zahlen zur Prävalenz entbehren jeglicher Evidenz

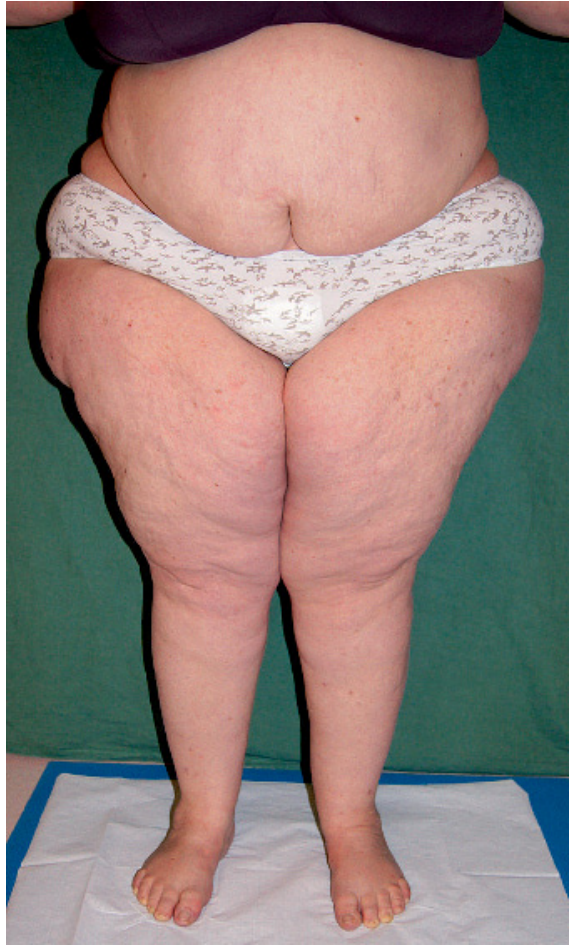

Abb. 1 Patientin mit Lipohypertrophie der Oberschenkel und der Beckenregion

und werden daher hier auch nicht aufgeführt (3).

Trotz des Fehlens objektiver Diagnosekriterien wird aber deutlich, dass die Beschwerdesymptomatik zwingend zur Diagnose Lipödem gehört. Die isolierte Disproportionalität des Frauenbeins - ohne o.g. Beschwerden - wird als Lipohypertrophie bezeichnet (4). Bei der Lipohypertrophie handelt es sich um eine anlagebedingte Fettgewebsvermehrung der Extremitäten. In manchen schweren Fällen kann hierdurch das Gangbild beeinträchtigt sein mit der Folge von orthopädischen Problemen. Die dann notwendige Behandlung unterscheidet sich aber von der des Lipödems.

- Abbildung 1 zeigt eine Patientin mit Lipohypertrophie der Oberschenkel und der Beckenregion. Die Patientin hat keine weiteren Beschwerden im Weichteilgewebe ihrer Beine; daher liegt auch kein Lipödem vor!

Das Lipödem entwickelt sich immer aus einer Lipohypertrophie heraus, allerdings nur bei einem sehr kleinen Teil der Betroffenen. Warum sich bei manchen Patientinnen (sehr selten sind auch Männer betroffen) eine Schmerzsymptomatik im Fettge- webe entwickelt und welche Pathophysiologie dieser Beschwerdesymptomatik zugrunde liegt, ist zum jetzigen Zeitpunkt noch unklar und Gegenstand aktueller Forschung (5-7).

Über das Lipödem kursieren zahlreiche Mythen; Statements, die Eingang in die Wissenschaft und damit auch Eingang ins „Patienten-Know-how" gefunden haben. Wir wollen in diesem Beitrag zwei dieser Mythen auf wissenschaftliche Evidenz untersuchen.

Ein Lipödem wurde von uns dann als gesichert gewertet, wenn die Patientinnen Beschwerden wie Druckschmerzhaftigkeit (oder Berührungsempfindlichkeit oder ein als belastend empfundenes ausgeprägtes Schweregefühl) sowie eine verstärkte Hämatomneigung im Bereich des überproportionalen Weichteilgewebe der Beine (oder der Arme) angaben. Gleichzeitig musste im Rahmen der klinischen Untersuchung der „Kneiftest“ positiv sein. Hierbei wird - gleichzeitig - eine Bauchfettfalte sowie eine Fettfalte im Bereich der Oberschenkel (und danach der Unterschenkel, ggf. auch Arme) moderat gekniffen. Die Lipödempatientin stellt dann - im Gegensatz zur Patientin mit reiner Lipohypertrophie - einen deutlichen Unterschied in der Schmerzwahrnehmung (Extremitätenschmerz) wahr. Der Nachweis eines dellenhinterlassenden Ödems war zur Diagnosesicherung nicht erforderlich, da ein relevantes Ödem beim Lipödem nach unserer klinischen Erfahrung ohnehin nur in sehr seltenen Fällen vorhanden ist. Dies Vorgehen entspricht auch den Diagnosekriterien der niederländischen sowie der britischen Lipedema Guidelines (8-10).

In unserer Darstellung über die Mythen des Lipödems fokussieren wir uns in diesem ersten Beitrag vor allem auf zwei Aspekte, die aufs Engste mit dem Lipödem verbunden sind: Auf die Adipositas, sowie auf die psychische Situation von Lipödempatientinnen - die wiederum eng mit der Adipositas in Zusammenhang steht.

In den folgenden Beiträgen wird die wissenschaftliche Evidenz weiterer populärer Lipödem-Statements diskutiert. Thema der nächsten Darstellung ist die Frage nach dem „Ödem im Lipödem" und damit auch die Rolle der Manuellen Lymphdrainage. 


\section{Mythos 1: Das Lipödem ist eine progrediente Erkrankung}

Dieses Statement findet sich in einer Vielzahl wissenschaftlicher Publikationen, ebenso wie auf Lipödemportalen im Internet sowie in Magazinen von LipödemSelbsthilfegruppen (11-14). Auch die aktuellen Deutschen S1-Lipödem-Leitlinien definieren das Lipödem als „progrediente Erkrankung“ (15), und das von Patienten häufig frequentierte Internetportal „Lipödem Hilfe Deutschland e.V. schreibt sogar: „Das Lipödem ist IMMER progredient, das bedeutet fortschreitend" (16 Hervorhebungen im Original).

Es ist unbestritten, dass manche Patientinnen eine massiv disproportionale Fettgewebsvermehrung - isoliert - im Bereich der Beine aufweisen. Doch bilden diese Patienten in unserem Patientengut eine sehr kleine Minderheit. Trotzdem dienen Bilder dieser kleinen Minderheit häufig als „typische“ Bilder von Lipödempatientinnen in Fach- und Laienpublikationen.

Der Terminus „progredient" suggeriert nun aber, dass sich dieses, in der Regel genetisch disponierte disproportionale Fettgewebe (17) - quasi autonom, schicksalhaft und unabhängig von der allgemeinen $\mathrm{Ge}$ wichtszunahme - vermehrt $(18,19)$. Diese Fettgewebsvermehrung verläuft dann in drei (in manchen Publikationen auch in vier) Stadien (20-22). Im NDR Gesundheitsratgeber liest man: „Fettzellen vermehren sich unkontrolliert" (23).

Doch wo ist die wissenschaftliche Evidenz für dieses pathophysiologische Konstrukt?

Daten, die die Progredienz des Lipödems bzw. eine diese Behauptung stützende Pathophysiologie belegen, existieren nicht! Gerne werden immer die Erstbeschreiber des Lipödems - E.V. Allen und E.H. Hines - bemüht, die in Ihrer 2. Publikation (gemeinsam mit E. Wold) aus dem Jahre 1951 erstmals den Terminus „Progressive enlargement of the limbs ..." benutzten (24). Allerdings sahen bereits die Erstbeschreiber, dass der progressive Verlauf des Lipödems üblicherweise mit $\mathrm{Ge}$ wichtszunahme verbunden ist („...is ordinarily associated with weight gain“). Diese
Gewichtszunahme beim Lipödem („gradual increase of body weight") wurde bereits in der Erstveröffentlichung aus dem Jahre 1940 betont (25).

Es drängt sich daher die Frage auf, ob der Umfang der Beine deshalb zugenommen hat, weil die Patientinnen- insgesamt - an Gewicht zugenommen haben. In diesem Falle wäre nicht das Lipödem progredient, sondern das Körpergewicht! Im Rahmen einer Gewichtszunahme würden dann erwartungsgemäß auch die Beinumfänge zunehmen. Für diese Sichtweise ist die Pathophysiologie offensichtlich!

Beim Blick auf unser Patientengut wird deutlich, dass ein enger Zusammenhang zwischen Übergewicht bzw. Adipositas und dem Krankheitsbild Lipödem vorliegt. In unserer kassenärztlichen lymphologischen Ambulanz sahen wir 2015 etwas über 2300 Patientinnen mit der Diagnose Lipödem. Nur $3 \%$ dieser Patientinnen waren normalgewichtig, $9 \%$ übergewichtig (BMI zwischen 25 und $30 \mathrm{~kg} \mathrm{~m} /{ }^{2}$ ) und $88 \%$ unserer Lipödempatientinnen waren adipös $\left(\mathrm{BMI}>30 \mathrm{~kg} / \mathrm{m}^{2}\right)$.

In diesem Zusammenhang ist zu erwähnen, dass der BMI bei Lipödempatientinnen im Übergewichtsbereich (selten auch noch im unteren Adipositasbereich) nur eingeschränkt verwertbar ist. Es gibt, wie oben dargestellt, die seltene Patientengruppe mit weitgehend schlankem Oberkörper und deutlicher Fettgewebsvermehrung der Extremitäten. Hier wird „formal“ Übergewicht vorgetäuscht, die durch die Fettgewebsverteilung aufgrund der Lipohypertrophie der Beine tatsächlich nicht gegeben ist. Für diese Patienten ist der WHtR (Waist-to-Height Ratio ,Taille-zuGröße-Verhältnis') das geeignetere Maß. Der WHtR bezeichnet das Verhältnis zwischen Taillenumfang und Körpergröße und gibt eine bessere Aussage zur Verteilung des Körperfettes. In der Földiklinik wird bei allen Patienten mit Lipödem sowohl der BMI als auch der WHtR ermittelt.

Die oben genannten Zahlen, die den engen Zusammenhang zwischen Adipositas und Lipödem darstellen, werden auch von Zentren in den Niederlanden sowie in Großbritannien bestätigt, die Lipödempatientinnen behandeln $(26,27)$. Die normalgewichtige Lipödempatientin ist eine Rarität!
Warum ist die Frage nach der Progredienz so wichtig, warum hat die Klärung dieses Statements eine so immense praktische Bedeutung?

Viele unserer Lipödem Patientinnen haben neben Ihren Beschwerden vor allem eines: sie haben Angst; Angst, dass ihr Lipödem progredient ist. Eine große Mehrheit unserer Patienten hat sich bereits über das Internet über das Lipödem vorinformiert (und wurde hierdurch fehlinformiert!). Dort werden oft Bilder von Patienten gezeigt, die eine extreme (in der Realität aber sehr selten auftretende) Fettgewebsvermehrung an Beinen oder Armen aufweisen. Hierdurch äußern die meisten unserer Patientinnen große Sorge, dass ihr Lipödem auch solche Ausmaße annehmen könnte. Aus diesem Grunde hat die Klärung dieser Frage eine große Bedeutung.

Abbildung 2 und Abbildung 3 zeigen Patientinnen mit der Diagnose Lipödem: Abbildung 2 mit ausgeprägter Fettgewebsvermehrung isoliert im Bereich der Oberschenkel, Abbildung 3 mit massiver Fettgewebsvermehrung im Bereich der Oberschenkel sowie der Unterschenkel. Die Füße sind beim Lipödem typischerweise unauffällig. Beide Patientinnen präsentieren Extrembefunde, die wir in unserer klinischen Praxis selten sehen. Die im Vordergrund stehende Erkrankung bei beiden Patientinnen ist aber - und das sei betont die morbide Adipositas!

Unsere alltägliche klinische Erfahrung ist jedoch eine ganz andere als das Behandeln dieser Extrembefunde. Wir sehen regelmäßig- stationär aber auch ambulant Lipödem-Patientinnen, die, wenn das Gewicht stabil geblieben ist, auch über viele Jahre ein stabiles Lipödem präsentieren. Inzwischen sehen wir hier 20 Jahres-Verläufe von Patientinnen mit stabilem - nicht progredienten - Lipödem; stabil, weil diese Patientinnen ihr Gewicht (auf unterschiedlichem Gewichtsniveau) stabilisiert haben.

Die Patientin auf $>$ Abbildung 4 wird von uns seit ca. 10 Jahren aufgrund ihres Lipödems ambulant mitbetreut. Der BMI dieser Patientin lag in dieser Zeit stabil um $31 \mathrm{~kg} / \mathrm{m}^{2}$, die WHtR um 0,53. Die Beinvolumina (Ober-und Unterschenkel getrennt vermessen) haben sich in diesen 10 Jahren praktisch nicht verändert. Unter täglichem Tragen der flachgestrickten Kompressions- 


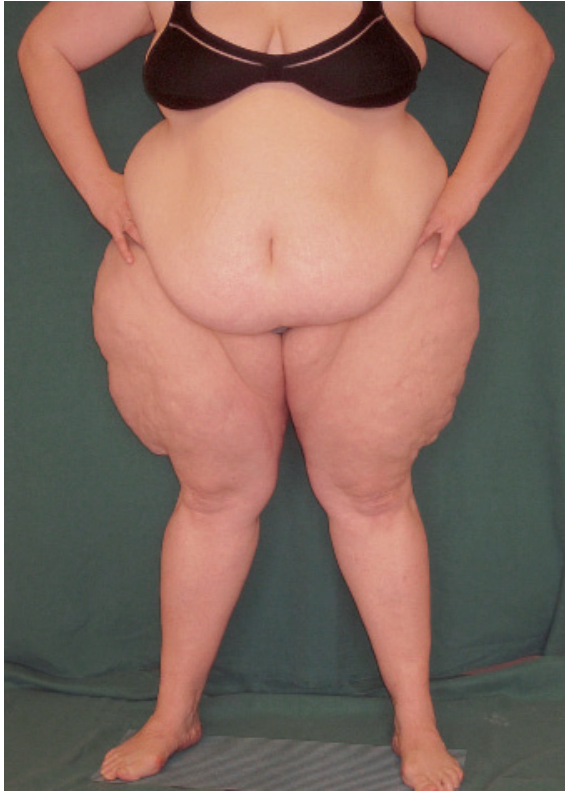

Abb. 2 Patientin mit der Diagnose Lipödem mit ausgeprägter Fettgewebsvermehrung isoliert im Bereich der Oberschenkel

strumpfhose sowie regelmäßig 2-3-mal wöchentlich durchgeführter sportlicher Aktivität ist die Patientin beschwerdefrei.

Allerdings - die Mehrheit unserer Patientinnen erfährt im Laufe von Jahren eine Gewichtsprogredienz. Diese kann kontinuierlich sein; sehr viel häufiger erleben $\mathrm{Pa}$ tientinnen mit Lipödem eine Gewichtszunahme, die durch regelmäßiges „Diäten“ und nachfolgendem Jo-Jo-Effekt auftritt.

Beim Lipödem ist der Rat konventionell Gewicht abzunehmen besonders tückisch. Auf der einen Seite nehmen 95\% aller Menschen, die mit den üblichen kommerziellen und nichtkommerziellen Diäten Gewicht abnehmen, binnen von 3 Jahren ihr Gewicht wieder zu (28-35). Gleichzeitig haben Studien der Universität Hohenheim ergeben, dass Frauen (im Gegensatz zum Männern) nach einer Gewichtsabnahme überproportional im Bereich der unteren Körperhälfte Gewicht zunehmen (36). Mit anderen Worten: mit jeder ärztlichen Empfehlung an die Lipödem-Patientin, Gewicht abzunehmen erhöht sich das Risiko, dass sich das Lipödem verschlechtert.

Es wird deutlich, dass es keine Evidenz dafür gibt, dass ein Lipödem progredient ist. Tatsächlich ist häufig das Körpergewicht progredient und - in Folge dessen -

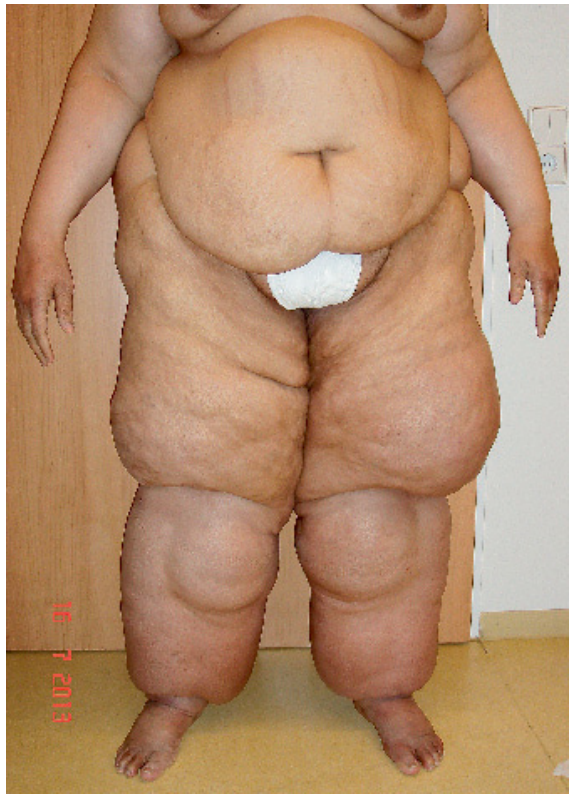

Abb. 3 Patientin mit der Diagnose Lipödem mit massiver Fettgewebsvermehrung im Bereich der Oberschenkel sowie der Unterschenkel

kommt es auch zu einer Verschlechterung des Lipödems.

Wenn das Lipödem aber nicht progedient ist, dann ist auch der Terminus „Lipolymphödem“ nicht sinnvoll. „Lipolymphödem“ suggeriert, dass das "progrediente Lipödem“ zum Lymphödem führt, es suggeriert, dass das Lipödem die Ursache des Lymphödemes ist. In manchen Klassifikationen wird das „Lipolymphödem" auch als Lipödem im Stadium IV bezeichnet. Karen Herbst von der University of Arizona und Protagonistin der amerikanischen Lipödemszene schreibt: „Lipedema therefore is a pre-lymphedema condition" (37).

Es muss betont werden, dass es für diese populäre Sichtweise keine ausreichende wissenschaftliche Evidenz gibt. Weder existieren histologische Untersuchungen, die das Konstrukt „Lipolymphödem“ stützen, noch gibt es bildgebende Verfahren, die einen entsprechenden Nachweis erbracht haben. Letztlich bezieht sich die große Mehrheit der Autoren bei Erwähnung des „Lipolymphödems" auf die Arbeiten von Amann-Vesti aus dem Jahre 2001 sowie von Bilancini et al. aus dem Jahre 1995 (38, 39). In beiden Studien wurden jeweils nur 12 (!) Patienten untersucht. Während Bilancini im Rahmen einer dynamischen

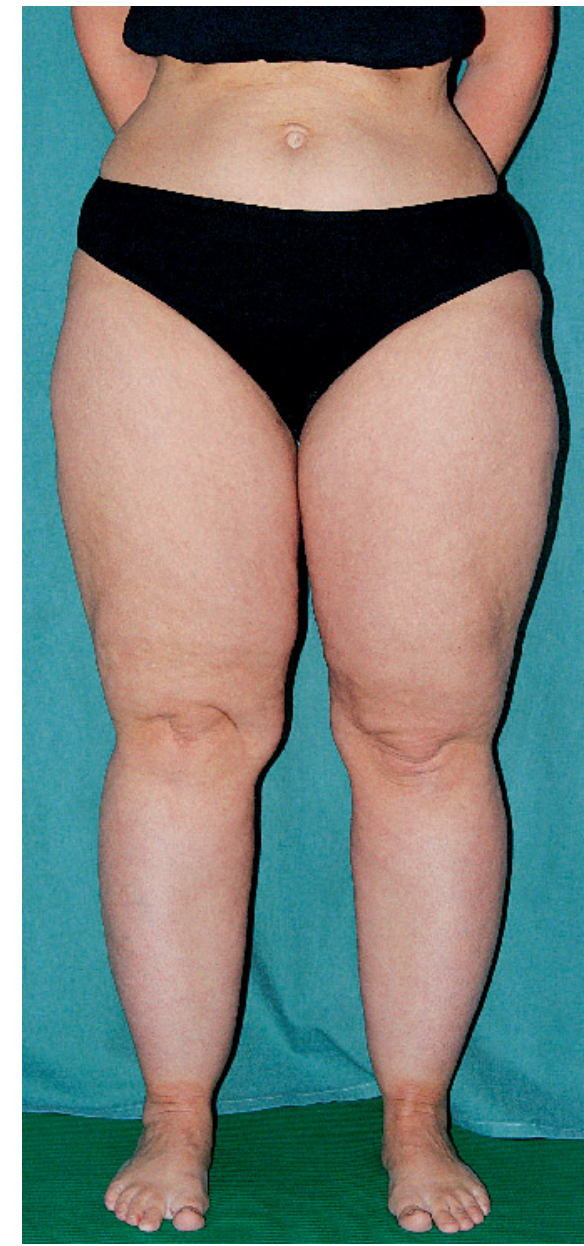

Abb. 4 Patientin, die von uns seit ca. 10 Jahren aufgrund ihres Lipödems ambulant mitbetreut wird.

Lymphszintigraphie einen verlangsamten Lymphfluss bei Lipödempatientinnen feststellte fand Amann-Vesti mittels Fluoreszenz-Mikrolymphograhie beim gleichen Patientengut die inzwischen häufig zitierten Mikroaneurysmen der Lymphkapillaren. Allerdings war die Transportfähigkeit des Lymphgefäßsystems bei Aman-Vesti in keiner Weise eingeschränkt. Auch weitere klinische Studien mittels indirekter Lymphographie und Lymphszintigraphie haben gezeigt, dass der Lymphtransport vom subepidermalen Kompartiment beim Lipödem im Gegensatz zum Lymphödem funktioniert (40-42).

Große Schwäche der Daten von Bilancini als auch von Amann-Vesti ist die fehlende Beschreibung der Gewichtssituation der untersuchten Patientinnen. In beiden $\mathrm{Pu}$ blikationen fehlen jegliche Angaben zum 
BMI der Patientinnen. Die Angabe des BMI wäre aber essenziell, um zu unterscheiden, ob die Auffälligkeiten im Lymphgefäßsystem tatsächlich - wie postuliert und vielfach zitiert - auf dem Boden des Lipödems zu sehen sind oder doch eher Adipositas-induziert. Amann-Vesti beginnt ihre Darstellung sogar mit der Feststellung "Lipedema is a special form of obesity“ (43). Es kann daher davon ausgegangen werden, dass die von ihr untersuchten Lipödempatientinnen adipös, manche vielleicht auch schwer adipös waren. Die Vermutung liegt daher nahe, dass die gesehenen Veränderungen der Lymphkapillaren Adipositas-induziert waren - im Sinne eines beginnenden Adipositas-assoziierten Lymphödems.

Unsere Erfahrung mit Tausenden von Lipödempatientinnen in den vergangenen Jahren unterstützt auch von klinischer Seite diese Vermutung. Wenn die $165 \mathrm{~cm}$ große und $90 \mathrm{~kg}$ schwere Lipödempatientin weitere 20 oder $40 \mathrm{~kg}$ Gewicht zunimmt, kann sich additiv zum Lipödem auch ein Lymphödem entwickeln. Dieses Lymphödem ist dann aber kein Lipödem-induziertes Lymphödem, sondern ein Adipositas-assoziiertes Lymphödem. Die Pathophysiologie hierzu wurde im Beitrag zum Adipositas-assoziieren Lymphödem in diesem Heft skizziert.

Der Terminus „Lipolymphödem“ sollte daher aus dem lymphologischen Sprachgebrauch getilgt werden. Er sollte auch deshalb getilgt werden, weil er häufig missbraucht wird, um die von Lipödempatientinnen meist begehrte Manuelle Lymphdrainage rezeptieren zu können. Dass dieser Missbrauch auch von offizieller Seite gefördert wird, erinnert schon stark an ein Possenstück. Die Manuelle Lymphdrainage wird im Heilmittelkatalog - zu Recht nicht als Indikation für das Lipödem aufgeführt. Dies macht medizinisch Sinn, da die Evidenz für ein relevantes Ödem (Ödem im Sinne von Flüssigkeit) beim Lipödem komplett fehlt. (Dieser Punkt ist Thema von Teil 2 dieser Darstellung). Diese -medizinisch vernünftige - Entscheidung wird allerdings in einem gemeinsamen Fragenund Antworten-Katalog der Spitzenverbände der Krankenkassen und der Kassenärztlichen Bundesvereinigung (KBV) durch folgende Formulierung ad absurdum

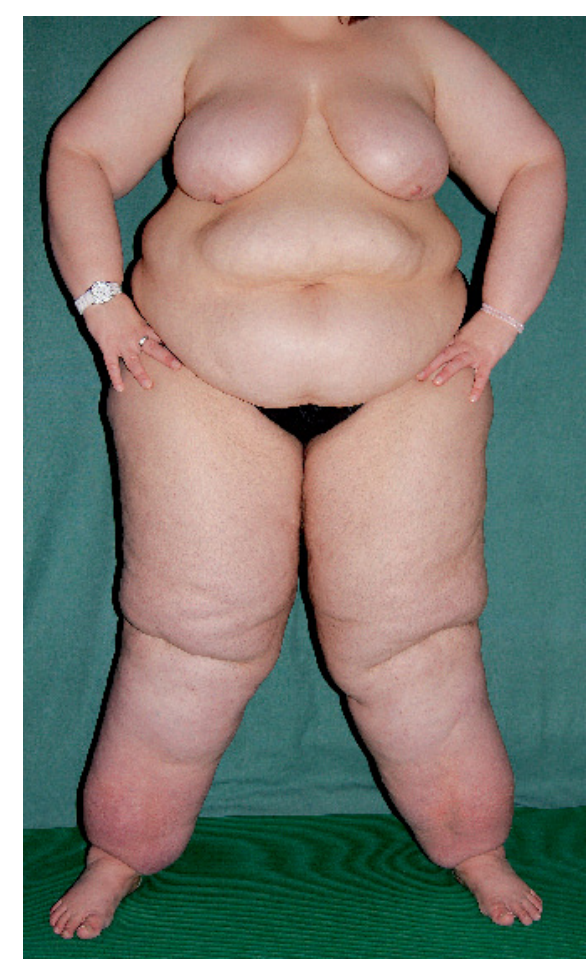

Abb. 5 Patientin, die über Jahre wegen eines Lipödems ambulant behandelt wurde.

geführt: „Das Lipoedem wird synonym auch Lipolymphoedem genannt; demnach ist eine Einordnung unter LY1 oder LY2 möglich und könnte mit einer MLD behandelt werden" (44).

Auf diesen per Verwaltungsakt offiziell legalisierten Unsinn wird natürlich auch in den Patientenforen und Selbsthilfegruppen ausführlich hingewiesen (45)!

Mit anderen Worten: hier werden zwei ganz unterschiedliche Erkrankungen (Lipödem und Lymphödem) miteinander vermischt, um die Verordnungsfähigkeit einer Therapie zu ermöglichen; einer Therapie, die für das eine Krankheitsbild (Lipödem) keinen nachgewiesenen Effekt hat, während sie für das andere Krankheitsbild (Lymphödem) ein zentrales Therapiemodul darstellt!

In unserer klinischen Praxis sehen wir meist Patientinnen, die diese drei Krankheitsbilder gleichzeitig präsentieren: Adipositas, Lymphödem sowie Lipödem! Bei diesen Patientinnen ist die Manuelle Lymphdrainage essenziell - aber nicht aufgrund des Lipödems, sondern aufgrund des Adipositas-assoziierten Lymphödems. - Abbildung 5 zeigt eine Patientin, die über Jahre wegen eines Lipödems ambulant behandelt wurde. In den vergangenen 8 Jahren kam es zu einer Gewichtszunahme von ca. $40 \mathrm{~kg}$. Inzwischen hat sich additiv zum Lipödem auch ein distal betontes Beinlymphödem entwickelt. Die empfohlene therapeutische Herangehensweise dieses Krankheitsbilds wird im Beitrag über die Adipositas-assoziierten Lymphödeme (in diesem Heft) dargestellt.

\section{Fazit}

Es gibt keine wissenschaftliche Evidenz, dass das Lipödem einen progredienten Verlauf hat! Vielmehr ist Gewichtszunahme, ist Adipositas sehr häufig progredient. Die WHO spricht bereits von einer Adipositasepidemie. Erst im Zuge der Gewichtsprogredienz kann es dann zu einer Verschlechterung des Lipödems kommen. Folglich ist auch der Terminus „Lipolymphödem“ aus medizinischer Sicht falsch!

Diese Sichtweise auf das Lipödem sollten wir als gute Nachricht begreifen, eine Nachricht, die wir mit unseren Lipödempatientinnen teilen sollten. Wir können unseren Patientinnen sagen, dass ihr Lipödem nicht fortschreitet, dass es sich nicht verschlechtert; dass es stabil bleibt - wenn auch ihr Gewicht stabil bleibt!

\section{Mythos 2: Ein Lipödem macht psychisch krank.}

Von einem Lipödem betroffene Frauen können unter einer Vielzahl an Problemen leiden. Dies betrifft vor allem das Erleben der Lipödem-assoziierten Schmerzen und Bewegungseinschränkungen, was zu einer physischen Minderung der Lebensqualität führt $(46,47)$. Zudem leiden viele Frauen mit Lipödem an der Unzufriedenheit mit der Disproportionalität ihres Körpers und damit verbundener Stigmatisierung. Sie haben Probleme mit der Akzeptanz des eigenen Körpers und der Selbstakzeptanz, sowie deren Folgen $(46,47)$. Häufig erfahren die Betroffenen selbst unter Ärzten zunächst Unverständnis für ihre Probleme (50); oft dauert es mehrere Jahre, bis die Diagnose Lipödem gestellt wird und eine geeignete Therapie erfolgt. 
Mehrfach wird in Veröffentlichungen über psychische Störungen bei Lipödempatientinnen berichtet (50-54). Insgesamt ist die Datenlage zur Beziehung von Lipödem und Psyche derzeit jedoch sehr dürftig. Eine Studie an 100 Lipödempatientinnen der Liposuktionsklinik von Stutz kommt zu dem Resultat, dass $74 \%$ an chronischen Essstörungen leiden (53), 8\% haben sogar mindestens einen Suizidversuch unternommen (54).

In einer von Smidt 2015 initiierten weltweit angelegten internetgestützten Befragung von $\mathrm{N}=1416$ Teilnehmerinnen gaben als Selbsteinschätzung 39,7\% Depression an - gegenüber einer Prävalenz von 3-17\% in der Allgemeinbevölkerung - 16,5\% Essstörungen - gegenüber einer Prävalenz von $1-5 \%$ in der Allgemeinbevölkerung. Allerdings bestand bei $30,37 \%$ der Teilnehmerinnen keine ärztlich gestellte Diagnose eines Lipödems (52)!

Eine ebenfalls internetbasierte Untersuchung von Dudek et al 2016 (51) an N=328 Teilnehmerinnen ergab in der Selbsteinschätzung bei 31,8\% Essstörungen. Depression und Angst wurden hier mittels Patients Health Questionnaire 9 diagnostiziert, wobei $56,8 \%$ der Teilnehmerinnen erhöhte bis stark erhöhte Depressionswerte aufwiesen.

Bei anderen somatischen Erkrankungen wie z.B. Tumorerkrankungen werden psychische Faktoren seit Jahren bei der Genese berücksichtigt (z.B. 55). So spielen z.B. auch bei rheumatoider Arthritis (56) psychische Faktoren wie Stress eine wichtige Rolle.

Bislang wird bezüglich Lipödem implizit der Eindruck erzeugt, als wären psychische Störungsbilder wie Depression oder Essstörungen ausschließlich die Folge des Lipödems. Aber - stimmt dies wirklich?

Generell zeigt sich die Problematik in der Forschung vor allem in zwei Bereichen: Erstens besteht die Gefahr, durch Reduktion der Komplexität wichtige Aspekte zu übersehen. Zweitens werden statistische Zusammenhänge im Sinne einer Korrelation oft fälschlicherweise als Kausalität interpretiert. So stellt sich die Frage, bei wie vielen Frauen mit Lipödem es wirklich das Lipödem ist, das kausal zur psychischen Störung führt, bei wie vielen es lediglich eine Komorbidität ist und bei wie vielen wo- möglich die psychische Störung eine wichtige Beteiligung an der Entstehung der Schmerzsymptomatik des Lipödems hat.

Um zu einer Klärung dieser offenen Fragen beizutragen, führten wir eine Pilotstudie in unserer Klinik durch.

\section{Pilotstudie}

Fragestellung: Führt ein Lipödem zu psychischen Störungen?

Methodik: Patientinnen, die im Studienzeitraum von April bis Dezember 2017 im Rahmen ihres stationären Aufenthaltes zufällig einer bestimmten Station der Földiklinik zugewiesen wurden, wurden in die Studie eingeschlossen. Bedingung war die ärztlich in der lymphologischen Fachklinik erneut gesicherte Diagnose eines Lipödems der Beine mit den typischen Symptomen der Disproportionalität, Hämatomneigung und Schmerzen, die auf das Lipödem zurückzuführen sind.

Im Folgenden wurden von einer speziell auch in diesem Patientengut erfahrenen Psychologischen Psychotherapeutin halbstrukturierte Interviews mit den Patientinnen geführt, die meist auf zwei Termine verteilt wurden. Diese umfassten:

1. die Erfassung der aktuell bestehenden psychischen Störungen nach ICD Kriterien (57) sowie im Lebensverlauf jene psychischen Störungen, die in der Vergangenheit bestanden hatten

2. die mit dem Lipödem verbundene Symptomatik (vgl. 58)

3. das gemeinsame Überblenden der beiden Bereiche.

Fragebögen stellten sich für den explorativen Charakter der Studie als ungeeignet heraus, da sie ein zu kleines Spektrum an

Tab. 1 Beschreibung der Stichprobe: $\mathrm{N}=45$

\begin{tabular}{l|l|l|l}
\hline & $\begin{array}{l}\text { Patientinnen mit } \\
\text { BMI }<40 \mathrm{~kg} / \mathrm{m}^{2} \\
\mathrm{n}_{1}=17\end{array}$ & $\begin{array}{l}\text { Patientinnen mit } \\
\text { BMl }>40 \mathrm{~kg} / \mathrm{m}^{2} \\
\mathrm{n}_{2}=28\end{array}$ & $\begin{array}{l}\text { Gesamtstichprobe } \\
\mathrm{N}=45\end{array}$ \\
\hline Alter in Jahren & $17-62$ & $32-62$ & $17-62$ \\
\hline Mittelwert Alter & 41,06 & 45,61 & 43,88 \\
\hline Range BMI in $\mathrm{kg} / \mathrm{m}^{2}$ & $24,01-39,48$ & $40,65-71,52$ & $24,01-71,52$ \\
\hline Mittelwert BMI & 31,47 & 48,39 & 41,99 \\
\hline
\end{tabular}

Symptomatik abbilden (vgl. 59-62), psychische Symptomatik bei somatischen Erkrankungen z.B. bei Depression überschätzen $(63,64)$ und den zeitlichen Verlauf nicht abbilden können. Gerade im Konzept der Depression zeigt sich neuerdings zudem, dass es sich dabei um kein einheitliches Konstrukt handelt, sondern verschiedene Symptomcluster bestehen $(65,66)$, deren Berücksichtigung für eine erfolgreiche psychotherapeutische Behandlung relevant ist.

\section{Ergebnisse}

Es wurden $\mathrm{N}=45$ Patientinnen in die Studie aufgenommen ( Tab. 1). Dargestellt werden hier die wichtigsten Ergebnisse, zur besseren Lesbarkeit im Text gerundet auf ganze Zahlen. Dabei wurden die Untergruppen $\mathrm{n} 1 \mathrm{mit}$ einem $\mathrm{BMI}<40 \mathrm{~kg} / \mathrm{m}^{2}$ und $\mathrm{n} 2$ mit einem $\mathrm{BMI} \geq 40 \mathrm{~kg} / \mathrm{m}^{2}$ unterschieden.

84\% der Gesamtstichprobe zeigen eine stark ausgeprägte psychische Symptomatik ( Tab. 2), die der Entwicklung Lipödemassoziierter Schmerzen vorausgeht. $\mathrm{Zu}$ sammengefasst wurden hier psychische Störungen sowie Symptome, die nur knapp den Ausprägungsgrad einer leichten depressiven Episode verfehlen, ebenso auch das Burnout-Syndrom (67). Letzteres gilt in der Diagnostik nicht als psychische Störung, sondern als leistungsbezogene Arbeits- und Motivationsstörung. Belastende Lebensereignisse, die nur kurzzeitig Stress und Belastung zur Folge haben, treten im Lebensverlauf bei fast allen teilnehmenden Frauen auf, zeigen in der Pilotstudie aber keine Auswirkungen auf die Schmerzsymptomatik des Lipödems.

Dabei zeigt sich, dass bei 53\% der Gesamtstichprobe $\left(\mathrm{n}_{1}: 47 \%, \mathrm{n}_{2}: 57 \%\right)$ zum 
Zeitpunkt der Untersuchung mindestens eine psychische Störung bestand. Bei den aktuellen Diagnosen der Studienteilnehmerinnen ergibt sich folgendes Bild $(>$ Tab. 3):

Depressive Störungen stehen im Vordergrund, wobei in dieser Kategorie nur jene gelistet sind, die zumindest den Ausprägungsgrad einer leichten depressiven Episode erreichen. Dysthymia wurde unter Sonstige eingeordnet. In der klinischen Diagnostik erfüllen 16\% das Kriterium einer Essstörung, bei 18\% besteht eine Auffälligkeit im Essverhalten, die noch nicht das Kriterium einer Essstörung nach ICD erreichen. $\mathrm{Zu}$ beachten ist auch, dass 7\% der Teilnehmerinnen bereits vor der Entwicklung eines Lipödems eine posttraumatische Belastungsstörung entwickelt hatten. Nur 4\% der Frauen mit Lipödem zeigten eine akzentuierte Persönlichkeit mit perfektionistischen Zügen - eine „Spielart“ der Normalität, die keine Persönlichkeitsstörung darstellt.

Von 16\% der Frauen der Gesamtstichprobe wurden konkrete Suizidgedanken in der Vergangenheit berichtet, wie z.B. von einer Brücke zu springen oder sich vor ei-

Tab. 2 Auftreten psychischer Störungen oder psychischer Vulnerabilitäten vor der Entwicklung eines Lipödems in \% der nach BMI eingeteilten Untergruppen

\begin{tabular}{l|l|l|l}
\hline $\begin{array}{l}\text { Psychische Störun- } \\
\text { gen oder Vulnerabili- } \\
\text { täten vor Lipödem }\end{array}$ & $\begin{array}{l}\text { Patientinnen mit } \\
\mathrm{BMI}<40 \mathrm{~kg} / \mathrm{m}^{2} \\
\mathrm{n}_{1}=\mathbf{1 7}\end{array}$ & $\begin{array}{l}\text { Patientinnen mit } \\
\mathrm{BMl}>\mathbf{4 0} \mathrm{kg} / \mathrm{m}^{2} \\
\mathrm{n}_{2}=\mathbf{2 8}\end{array}$ & $\begin{array}{l}\text { Gesamtstichprobe } \\
\mathrm{N}=\mathbf{4 5}\end{array}$ \\
\hline ja & 94,12 & 78,57 & 84,44 \\
\hline nein & 5,88 & 21,43 & 15,56 \\
\hline
\end{tabular}

Tab. 3 Aktuelle psychische Störungen (ohne sonstige psychische Vulnerabilität) in \% der nach BMI eingeteilten Untergruppen bzw. in \% der Gesamtstichprobe $\mathrm{N}=45$, teilweise mit Mehrfachdiagnosen

\begin{tabular}{l|l|l|l}
\hline & $\begin{array}{l}\text { Patientinnen mit } \\
\mathrm{BMI}<\mathbf{4 0} \mathrm{kg} / \mathrm{m}^{\mathbf{2}} \\
\mathrm{n}_{1}=\mathbf{1 7}\end{array}$ & $\begin{array}{l}\text { Patientinnen mit } \\
\mathrm{BMI}>\mathbf{4 0} \mathrm{kg} / \mathrm{m}^{\mathbf{2}} \\
\mathrm{n}_{2}=\mathbf{2 8}\end{array}$ & $\begin{array}{l}\text { Gesamtstichprobe } \\
\mathrm{N}=\mathbf{4 5}\end{array}$ \\
\hline Depressive Störungen & $\mathbf{2 3 , 5 3}$ & 39,29 & 33,33 \\
\hline Essstörungen & 5,89 & 21,43 & 15,56 \\
\hline Angststörungen & 5,89 & 10,74 & 8,89 \\
\hline $\begin{array}{l}\text { Posttraumatische } \\
\text { Belastungsstörungen }\end{array}$ & 5,89 & 7,14 & 6,67 \\
\hline $\begin{array}{l}\text { Schmerzen mit } \\
\text { somatoformen Anteilen }\end{array}$ & 11,76 & 3,57 & 6,67 \\
\hline Sonstige & 5,89 & 7,14 & 6,67 \\
\hline
\end{tabular}

ne Beteiligung der Lipödem-Beschwerden an der Entstehung einer psychischen Störung festzustellen. Bei $89 \%$ standen die nach einem Lipödem neu aufgetretenen psychischen Störungen in keinem direkten Zusammenhang zu den Beschwerden des Lipödems.

\section{Diskussion}

Die Ergebnisse zeigen, dass im klinischen Interview genauere diagnostische Daten erhoben werden können als in den bisherigen Online Befragungen. Die spontane Selbsteinschätzung der Patientinnen in Fragebögen überschätzt das Bestehen einer psychischen Störung oft. Viele Bereiche können im Interview differenzierter erfragt werden. Während in Online Befragungen $(68,69)$ „unerklärbare Gewichtszunahme“ von 56,7\% der befragten Frauen angegeben wurde, wurden im Interview der vorliegenden Pilotstudie stets - manchmal auch schambesetzt - verschiedene Gründe für eine Gewichtszunahme genannt. Dies zeigt die Vorteile von strukturierten Interviews, auch wenn sie einen hohen Zeitaufwand erfordern.

Es bestanden bei den meisten Frauen mit Lipödem bei bereits im Vorfeld der Entwicklung Lipödem-assoziierter Schmerzen stark ausgeprägte psychische Symptome über einen mehrmonatigen Zeitraum. Rein formallogisch - und dies ist gesichert - kann das, was zeitlich der Entwicklung eines Lipödems vorausgeht, nicht die Folge davon sein.

Die Ergebnisse sprechen klar dafür, dass ein Lipödem nur in seltenen Fällen direkt zu psychischen Störungen führt und die bisherige Sichtweise um die Vorgeschichte erweitert werden muss. Das Statement „ein Lipödem macht psychisch krank “ ist für die große Mehrheit der Frauen mit Lipödem schlichtweg falsch. Sind die somatischen Voraussetzungen zur Entwicklung eines Lipödems vorhanden, müsste es stattdessen heißen: „Es spricht vieles dafür, dass psychische Faktoren wesentlich zur Entwicklung eines Lipödems beitragen können“.

Um klar ursächliche Zusammenhänge herauszuarbeiten, wären Interviews einer größeren Kohorte wünschenswert, sowie der Einsatz einer geeigneten Kontrollgrup- 
pe, z.B. Frauen mit einer Lipohypertrophie - einer Fettgewebsvermehrung am schmerzfreien Bein. Da hier kein Behandlungsauftrag besteht, sehen wir solche Frauen aber in der Fachklinik fast nie.

Die beiden Krankheitsbilder Lipödem und Adipositas können nicht unabhängig voneinander untersucht werden: $82 \%$ der Teilnehmerinnen an der Pilotstudie sind adipös. Dies entspricht in etwa der Quote der Földiklinik: Wie bereits oben erwähnt, waren 88\% der mehr als 2300 in der Földiklinik ambulant wegen eines Lipödems behandelten Patientinnen adipös. Aber auch in den bisherigen Online Befragungen ist der prozentuale Anteil adipöser Frauen mit Lipödem hoch: z.B. 46\% (N=120) mit einem BMI $>40 \mathrm{~kg} / \mathrm{m}^{2}$ (6), oder bei einer weiteren Studie ein mittlerer BMI von $41,25 \mathrm{~kg} / \mathrm{m}^{2}(\mathrm{~N}=321)(51)$.

Wie von Luppino (70) in einer Metaanalyse dargestellt, besteht zwischen Adipositas und Depression ein bidirektionaler Zusammenhang. So erhöht das Auftreten von Depression das Risiko für Adipositas um 58\%, das Auftreten von Adipositas das Risiko, depressiv zu werden, um 55\%. Berücksichtigt man die Tatsache, dass eine Gewichtszunahme zur Verschlechterung des Lipödems führen kann dann ist es nicht verwunderlich, dass eine stark ausgeprägte psychische Symptomatik oft auch begleitet von Gewichtszunahme der Entwicklung von Lipödem-assoziierten Schmerzen vorausgeht.

Dass psychische Faktoren die Wahrnehmung und Verarbeitung von Schmerzen modulieren, ist inzwischen hinreichend belegt (71). Bislang existieren keine Untersuchungen zu Schmerzen beim Lipödem, jedoch einige zu Schmerzen bei orthopädischen (72) oder rheumatologischen Erkrankungen (56). Als negative Einflussfaktoren auf die Schmerzintensität wirken Rückzugsverhalten, Hilf- und Hoffnungslosigkeit, traurig niedergeschlagene Stimmung (71) oder katastrophisierendes Denken (72). Die Zusammenhänge zwischen psychischen Faktoren und Schmerzerleben sind dabei bidirektional.

Vielfach beginnt die grundlegende Problematik bereits deutlich vor der Entwicklung einer psychischen Störung oder eines Lipödems. Ein Schönheitsideal, das Frauen mit Normalgewicht und Konfektionsgröße
38 bereits als „Plus Size“ beschreibt (stellvertretend für einige: 73,74), lässt viele Frauen die Unzufriedenheit mit ihrem eigenen Körper spüren. Abnehmversuche durch zahlreiche Diäten - meist gefolgt von einem Jo-Jo-Effekt - münden bei Frauen mit entsprechender Disposition in einen Teufelskreis mit Verschlechterung der Disproportionalität.

Bereits die ersten und viel zitierten Arbeiten zum Lipödem von Allen und Hines 1940 (25) und 1951 (24) weisen auf die Bedeutung des Schönheitsideals hin: „Indeed, it is quite probable that much or all of the distress (both emotional and physical) associated with lipedema would not occur were "fat legs" considered a manifestation of beauty." (24).

\section{Fazit}

Das Lipödem ist keine psychische Störung, sondern eine somatische Erkrankung. Aktuell spricht jedoch einiges dafür, dass psychische Faktoren bei der Wahrnehmung Lipödem-bedingter Schmerzen eine entscheidende Rolle spielen können.

\section{Ausblick}

Neben den beiden dargestellten Mythen gibt es weitere, die sich um das Lipödem ranken, und die dringend einer Überprüfung bedürfen, deren Besprechung aber den Rahmen dieser Darstellung gesprengt hätte. Diese werden in weiteren Ausgaben dieser Zeitschrift diskutiert.

Beispielhaft hier erwähnt sei die Annahme, dass das Lipödem ein „Ödemproblem“ und Manuelle Lymphdrainage daher auch ein essenzieller und regelmäßig durchzuführender Therapiebestandteil sei. Populär ist auch das Statement, dass Gewichtsabnahme keinen Einfluss auf das Lipödem habe.

Am Ende dieser kleinen Lipödemreihe soll ein multimodales Behandlungskonzept zur Behandlung des Lipödems vorgestellt werden, ein Behandlungskonzept, dass zu einer nachhaltigeren und umfassenderen Beschwerdebesserung unserer Patienten beiträgt.
Interessenkonflikt

Nach Angaben der Autoren bestehen keine Interessenkonflikte.

\section{Ethische Richtlinien}

Für das Manuskript wurden keine Studien an Menschen oder Tieren durchgeführt.

\section{Literatur}

1. Reich-Schupke S et al. Dicke Beine - Nicht immer ist es ein Lipödem. JDDG 1610-0379.2013 225.

2. S 1 Leitlinie Lipödem S. 7. Verfügbar unter http://www.awmf.org/uploads/tx_szleitli nien/037-0121_S1_Lipoedem_2016-01.pdf

3. S 1 Leitlinie Lipödem S. 3 a.a.O.

4. Herpertz U. Ödeme und Lymphdrainage: Diagnose und Therapie - Lehrbuch der Ödematologie, 2. Aufl. Stuttgart: Schattauer 2013.

5. Brenner E. Wie kommt der Schmerz ins Lipödem. LymphForsch 2017; 21 (1): 40-47.

6. Lontok E. Lipedema- A giving smarter guide. Verfügbar unter http://assets1b.milkeninstitute.org/ assets/Publication/ResearchReport/PDF/Lipedema-Giving-Smarter-Guide.pdf

7. 6. Lipedema Foundation . Abrufbar unter https://www.lipedema.org

8. Lipedema Guidelines in the Netherlands 2014. Abrufbar unter https://diseasetheycallfat.tv/wp-content/uploads/2015/08/Dutch-lipoedema-guideline-2014.pdf

9. Halk AB, Damstra, RJ. First Dutch guidelines on lipedema using the international classification of functioning, disability and health. Phlebology $0(0)$ 1-8. 2016 Reprints and permissions: sagepub.co.uk/journalsPermissions.nav

10. Best Practise Guidelines Lipoedema UK 2017. Abrufbar unter http://www.lipoedema.co.uk/uk-bestpractice-guidelines/

11. Verein zur Förderung der Lymphödemtherapie e.V. Abrufbar unter http://www.lymphverein.de/li poedem.html

12. Meier-Vollrath I et al. Lipödem: Verbesserte Lebensqualität durch Therapiekombination Dtsch Arztebl 2005; 102(15): A-1061

13. Wagner S. Lymphedema and lipedema - an overview of conservative treatment. Vasa 2011 Jul;40(4): 271-279.

14. Wiedner $M$ et al. Entstehung des Lipödems. Lymph-Selbsthilfe 2017 (2) 15-16.

15. S 1 Leitlinie Lipödem S. 2 a.a.O

16. Lipödemhilfe e.V. Abrufbar unter http://www.li poedem-hilfe-ev.de/index.php/krankheit-lipoedem

17. Child A, Gordon C et al. Lipedema: an inherited condition. American J Med Genet . 2010; 152A(4): 970-976.

18. Bae Wook Shin et al. Lipedema, a Rare Disease. Ann Rehabil Med. 2011 Dec; 35(6): 922-927

19. Buck W, Herbst KL. Lipedema: A Relatively Common Disease with Extremely Common Misconceptions. Plast Reconstr Surg Glob Open 2016; $4(9)$. 
20. Dadras M et al. Liposuction in the Treatment of Lipedema: A Longitudinal Study. Arch Plast Surg. 2017; 44(4): 326.

21. Schmeller W. Meier-Vollrath I. Das Lipödem: neue Möglichkeiten der Therapie. Schweiz Med Forum 2007; 7: 151 .

22. Herbst KL et al. Lipedema Fat and Signs and Symptoms of Illness, Increase with advancing Stages. 2014. Abrufbar unter http://www.archive sofmedicine.com/medicine/lipedema-fat-andsigns-and-symptoms-of-illness-increase-with-advancing-stage.php?aid=674.

23. Norddeutscher Rundfunk Gesundheit Ratgeber. Was tun bei einem Lipödem. Abrufbar unter https://www.ndr.de/ratgeber/gesundheit/Was-tunbei-einem-Lipoedem,lipoedem 104.html

24. Wold LE Hines EA Allen EV: Lipedema oft he legs; a syndrome characterized by fat legs and edema. Ann Intern Med. 1951; 34(5): 1243-1250.

25. Allen E Hines E. Lipedema oft the legs: a syndrome characterized by fat legs and orthostatic edema. Proc Staff Mayo Clin 1940; 15: 184-187.

26. Child A, Gordon C et al. Lipedema: an inherited condition a.a.O.

27. Bosman J. Lipoedema: poor knowledge, neglect or disinterest. Journal of Lymphoedema 2011; 6 (2) 111.

28. Stunkard A. The Results of Treatment for Obesity. A Review of the Literature and Report of a Series. AMA Arch Intern Med 1959; 103(1): 79-85.

29. Bennett W, Gurin J.. The Dieter's Dilemma: Why Diets Are Obsolete-the New Setpoint Theory of Weight Control. New York: Basic Books 1982.

30. Cogan J, Rothblum E. Outcomes of weight-loss Programms. Genetic, Social and General Psychology Monographs 1993; 118; 4.

31. Perri MG et al. Sucess and failure in the treatment of obesity: Where do we go from here? Medicine, Exercise, Nutrition and Health 1995; 4: 255-272.

32. Hensrud DD, Weinsier RL. A prospective study of weight maintenance in obese subjects reduced to normal body weight without weight-loss training .American clinical Nutrtion 1994; 60(5): 688-694.

33. Mann T, Tomiyama AJ et al. Medicare's search for effective obesity treatments: diets are not the answer. Am Psychol 2007; 62(3): 220-233.

34. Nordmann A et al. Effects of low carb vs low fat diets on weight loss and cardiovascular risk factors: a meta analysis of randomized controlled trials. Arch Internal Med 2006166 (8).

35. Fildes A, Charlton J. Probability of an obese person attaining normal body weight. American Journal of Public Health 2015.

36. Bosy-Westhpal A. Effect of weight loss and regain on adipose tissue distribution, composition of lean mass and resting energy expenditure in young overweight and obese adults. International Journal of Obesity 2013; 1-7.

37. Herbst KL. Obesity and Lipedema - What's the link? Abrufbar unter http://www.obesityaction. org/wp-content/uploads/Lipedema_and_Obesity_online.pdf

38. Aman-Vesti BT et al. Microlymphatic aneurysms in patients with lipedema. Lymphology 2001 Dec;34(4): 170-175.
39. Bilancini S, Lucchi M, Tucci S et al. Functional lymphatic alterations in patients suffering from lipedema. Angiology 1995; 46: 333-339.

40. Harward CA et al. Lymphatic and venous function in lipoedema. Br J Dermatol 1996; 134(1): 1-6.

41. Bräutigam P, Földi E et al. Analysis of lymphatic drainage in various forms of leg edema using two compartment lymphoscintigraphy. Lymphology 1998; 31(2): 43-55.

42. Amann-Vesti BT et al. Druckmessung in den initialen Lymphgefäßen der Haut bei Patienten mit Lipödem. LymphForsch 2002; 6 (1): 7-9.

43. Aman-Vesti BT et al. Microlymphatic aneurysms in patients with lipedema a.a.O. S.170

44. Konsentierter Fragen-/Antwortenkatalog der Spitzenverbände der Krankenkassen und der Kassenärztliche Bundesvereinigung zu den HeilmittelRichtlinien nach $₫ 92$ SGB V mit Inkraftsetzung zum 1. Juli 2004. 11. Abrufbar unter http://www. kbv.de/media/sp/HeilM_RL_Aenderungen_FAQ.pdf

45. Lipödemportal. Abrufbar unter http://www.li poedemportal.de/lipoedem-informationen-fuertherapeuten.htm

46. Frambach Y Baumgartner A Schmeller W. Lipödem und Lebensqualität. Vasomed 2015; 27(5): 248-249.

47. Frambach Y Baumgartner A Schmeller W. Lipödem - eine „schwere“ Diagnose? Vasomed 2016; 5 $2-3$.

48. Dudek J. Psychological aspects of living with lipoedema. Svenska Ödemförbundet 10.06.2016. Abrufbar unter: https://youtu.be/R3ia7og9oEY

49. Dudek JE Bialaszek W Ostaszewski P. Quality of life in women with lipoedema: a contextual behavioral approach. Quality of Life Research 2016; 25: 401-408.

50. Fetzer A, Fetzer S. Lipoedema UK big survey 2014 Research Report. Lipoedema UK July 2016. www.li poedema.co.uk

51. Dudek J. Quality of Life and Psychological Functioning of Patients with Lipedema and Dercum's Disease. FDRS Goals forthe Future, Tools for Today. Salt Lake City 2017. fatdisorders.org. Abrufbar unter: https://youtu.be/xV-IVc0eVQM

52. Smidt T. Verfügbar unter www.tillysmidt.nl

53. Kraus RH. Alles über das Lipödem. Lymphologischer Informationsdienst. Abrufbar unter: http://www.lipoedemportal.de/lipoedem-folgeer scheinungen.htm

54. Stutz J. Understanding the Physical and Emotional Effects of Lipedema. Fat Disorders Research Society 2015; Abrufbar unter: https://youtu.be/ FI2RIRsZX0M

55. Grossarth-Maticek R. Krebsrisiken - Überlebenschancen. Heidelberg: Carl Auer Systeme 1998

56. Baerwald C. Einfluss von geringem und schwerem Stress auf eine Rheumaerkrankung. Deutsche Rheuma Liga. Abrufbar unter: https://www.rheu ma-liga.de/stress/

57. Dilling $\mathrm{H}$ Mombour W. Internationale Klassifikation psychischer Störungen: ICD-10 Kapitel V (F) - Klinisch-diagnostische Leitlinien. Hogrefe 2015 .
58. Schmeller W Meier-Vollrath I. Schmerzen beim Lipödem. Versuch einer Annäherung. LymphForsch 2008; 12: 7-14.

59. Carr T Moss T Harris D. The DAS24: A short form of the Derriford Appearance Scale DAS59 to measure individual responses to living with problems of appearance. Br J Health Psychol 2005 May 10 (Pt2) 285-298. Version of Record online: 31 DEC 2010 DOI: 10.1348/135910705X27613

60. Hilbert A, Tuschen-Caffier B. Eating Disorders Questionnaire. Deutschsprachige Übersetzung. Münster: Verlag für Psychotherapie 2006.

61. Löwe B, Zipfel S, Herzog W. Deutsche Übersetzung und Validierung des Brief Patient Health Questionnaire (Brief PHQ). Medizinische Universitätsklinik Heidelberg.

62. Maier W, Philipp M. Reliabilität und Validität der Subtypisierung und Schweregradmessung depressiver Syndrome. Berlin Heidelberg: Springer 2013.

63. Beck AT, Brown GK, Steer RA. Beck-DepressionsInventar-FS (BDI-FS). Manual. Deutsche Bearbeitung von Sören Kliem \& Elmar Brähler. Frankfurt am Main: Pearson Assessment 2013.

64. Hautzinger M, Keller F, Kühner C. BDI-II. BeckDepressions-Inventar. Revision. 2. Auflage. Frankfurt: Pearson Assessment 2009.

65. Fried EI, Nesse RM. Depression is not a consistent syndrome: an investigation of unique symptom patterns in the STAR ${ }^{*} \mathrm{D}$ study. Journal of Affective Disorders. 2015; 172: 96-102.

66. Fried EI, Epskamp S, Nesse RM. What are "good" depression symptoms? Comparing the centrality of DSM and non-DSM symptoms of depression in a network analysis. Journal of Affective Disorders. 2015; 189: 314-320.

67. Bauer J. Arbeit: Warum unser Glück von ihr abhängt und wie sie uns krank macht. Blessing 2013.

68. Schubert N Viethen H. Lipödem und Liplymphödem- Alles eine Frage des Lebensstils? LymphForsch 2016; 20:18-27.

69. Wilting J, Miller A. Kommentar zur Umfrage von Nadine Schubert und Heike Viethen: Lipödem und Liplymphödem - Alles eine Frage des Lebensstils? LymphForsch 2016; 20: 28-30.

70. Luppino FS. Overweight, Obesity, and Depression - A Systematic Review and Meta-analysis of Longitudinal Studies. Arch Gen Psychiatry. 2010; 67(3): 220-229.

71. Klinger R. Psychische Schmerzmodulation. Der Schmerz 2017 (2): 91-92.

72. Briest J, Bethge M. Der Einfluss von Katastrophisieren auf den Effekt von Depressivität auf Schmerz und körperliche Funktion. Der Schmerz 2017(2): 159-166. Abrufbar unter: https://doi.org/10.1007/s00482-016-0172-Z

73. Paragon Model Agentur. Abrufbar unter: https://paragonmodels.com/de/model/weiblich/ plus-size.html

74. Erdbeerlounge. Abrufbar unter: http://www.erd beerlounge.de/fashion/fashion-week/fw-berlin/ plus-size-model-masse-hat-man-ab-groesse-38/ 